\title{
Free tyrosine and tyrosine-rich peptide-dependent superoxide generation catalyzed by a copper-binding, threonine-rich neurotoxic peptide derived from prion protein
}

\author{
Ken Yokawa, Tomoko Kagenishi, Kaishi Goto, Tomonori Kawano ${ }^{凶}$ \\ Graduate School of Environmental Engineering, The University of Kitakyushu, Kitakyushu 808-0135, Japan.
}

Correspondence to: Tomonori Kawano, Ph.D., Department of Bioengineering, Graduate School of Environmental Engineering, The University of Kitakyushu, Kitakyushu 808-0135, Japan Tel: +81-93-695-3207. E-mail: kawanotom@env.kitakyu-u.ac.jp

Received: 2008.10.08; Accepted: 2008.12.29; Published: 2008.12.30

\begin{abstract}
Previously, generation of superoxide anion $\left(\mathrm{O}_{2}{ }^{*}\right)$ catalyzed by Cu-binding peptides derived from human prion protein (model sequence for helical Cu-binding motif VNITKQHTVTTTT was most active) in the presence of catecholamines and related aromatic monoamines such as phenylethylamine and tyramine, has been reported [Kawano, T., Int J Biol Sci 2007; 3: 57-63]. The peptide sequence (corresponding to helix 2) tested here is known as threonine-rich neurotoxic peptide. In the present article, the redox behaviors of aromatic monoamines, 20 amino acids and prion-derived tyrosine-rich peptide sequences were compared as putative targets of the oxidative reactions mediated with the threonine-rich prion-peptide. For detection of $\mathrm{O}_{2}{ }^{--}$, an $\mathrm{O}_{2}{ }^{-}$-specific chemiluminescence probe, Cypridina luciferin analog was used. We found that an aromatic amino acid, tyrosine (structurally similar to tyramine) behaves as one of the best substrates for the $\mathrm{O}_{2}{ }^{--}$generating reaction (conversion from hydrogen peroxide) catalyzed by Cu-bound prion helical peptide. Data suggested that phenolic moiety is required to be an active substrate while the presence of neither carboxyl group nor amino group was necessarily required. In addition to the action of free tyrosine, effect of two tyrosine-rich peptide sequences YYR and DYEDRYYRENMHR found in human prion corresponding to the tyrosine-rich region was tested as putative substrates for the threonine-rich neurotoxic peptide. YYR motif (found twice in the Y-rich region) showed 2- to 3-fold higher activity compared to free tyrosine. Comparison of $Y$-rich sequence consisted of $\mathrm{I} 3$ amino acids and its $\mathrm{Y}$-to- $\mathrm{F}$ substitution mutant sequence revealed that the tyrosine-residues on $\mathrm{Y}$-rich peptide derived from prion may contribute to the higher production of $\mathrm{O}_{2}{ }^{-}$. These data suggest that the tyrosine residues on prion molecules could be additional targets of the prion-mediated reactions through intra- or inter-molecular interactions. Lastly, possible mechanism of $\mathrm{O}_{2}{ }^{-}$generation and the impacts of such self-redox events on the conformational changes in prion are discussed.
\end{abstract}

Key words: Prion protein, Tyrosine, Superoxide, Redox, Copper

\section{Introduction}

Prion proteins (PrPs) found in animal brains showing transmissible spongiform encephalopathies (TSE) partially becomes proteinase K-resistant while those found in uninfected brains are sensitive to di- gestion by proteinase K [1]. The former form of PrPs represents the infectious scrapie isoform $\left(\mathrm{PrPsc}^{\mathrm{Ps}}\right)$ and the latter form of PrP represents the intrinsic cellular PrP (PrPC). Such deposition of abnormal protein fi- 
brils is a prominent pathological feature of many different "protein conformational" diseases, including prion dementias, Alzheimer's disease, Parkinson's disease and motor neuron disease [2]. In the cases of $\beta$-amyloid accumulation in Alzheimer's disease and of a-synuclein accumulation in Parkinson's disease, evidence for involvement of reactive oxygen species (ROS) such as $\mathrm{H}_{2} \mathrm{O}_{2}$ and hydroxyl radicals ( $\mathrm{HO}^{*}$ ) in the toxic mechanisms have been documented, suggesting that fundamental molecular mechanisms underlying the pathogenesis of cell death in neurodegenerative diseases could be attributed to the production of ROS that stimulates the formation of abnormal protein aggregates $[2,3]$.

Earlier studies have propounded the importance of metals (especially copper) in neurobiology and proposed two opposing roles for copper-bound PrPs as anti-oxidants required for the neuronal functions [4-6] and also as the pro-oxidants enhancing the neurodegenerative process [7]. The likely factors associated with generation [8] or removal of ROS [5] within PrPs are $\mathrm{Cu}$-binding sequences highly preserved in PrPs. In general, PrPs from mammals have six to seven putative $\mathrm{Cu}$-binding sites consisted of 4 distinct sequences (Fig. 1A), namely, PHGGGWGQ (octarepeat sequence, repeated for 4 times), GGGTH (existing just after the octarepeats), KTNMKHMA (known to be a portion of neurotoxic region), and VNITIKQHTVTTTT (helix 2, threonine-rich toxic sequence). The key common feature among the Cu-binding sequences in PrPs is the presence of His-residue(s) possibly behaving as the anchors for the copper $[9,10]$. The neurotoxic threonine-rich region corresponding to the human PrP helix 2 of the globular PrP domain is a good candidate to be one of the several 'spots' of intrinsic structural flexibility, which might induce local destabilization and concur to protein transformation, leading to aggregation-prone conformations [11].

Some in vitro studies with electron spin resonance spectroscopy have demonstrated that $\mathrm{H}_{2} \mathrm{O}_{2}$ (detected with a spin trap after conversion to $\mathrm{HO}^{\bullet}$ via Fenton reaction) is produced during the early stages of protein aggregation associated with neurodegenerative diseases involving $\beta$-amyloid, $\alpha$-synuclein and toxic forms of PrP [12-14]. Especially Cu-loaded PrP 106-126, the neurotoxic region, likely catalyzes the robust generation of $\mathrm{H}_{2} \mathrm{O}_{2}$ [14]. It is generally accepted that, like amyloid- $\beta$ peptide, prion induces apoptotic cell death in the neuronal tissues [15]. A PrP amino acid sequence 106-126 covering a putative Cu-binding site, likely forms the region causing neuronal apoptosis since a synthetic $\mathrm{Cu}$-bound peptide corre- sponding to $\operatorname{PrP} 106-126$ is highly fibrillogenic and highly toxic to neuronal cells as if behaving as a PrPsc mimic $[15,16]$.

In addition to the formation of $\mathrm{H}_{2} \mathrm{O}_{2}$ catalyzed by $\mathrm{Cu}$-loaded PrP peptides, Kawano [8] has reported that production of superoxide anion $\left(\mathrm{O}_{2}{ }^{-}\right)$, another key member of ROS in biological systems, is catalyzed by Cu-binding PrP fragments (corresponding to the octa-repeat, neurotoxic sequence and His-containing helical regions) in the presence of certain co-factors and aromatic monoamines known to behave as precursors and/or analogs of catecholamine-type neurotransmitters highly rich in brains. Based on the peroxidative model redox mechanism [9] in which $\mathrm{Cu}$-bound forms of PrP catalyzes the generation of ROS in the presence of some natural co-factors and inducers in the neuronal tissues, Kawano [8] has proposed that aromatic monoamines may form a group of ROS inducers (substrates) possibly contributing to the PrP-dependent oxidative burst. Earlier reports by Japanese team are in support of such view, showing that $\mathrm{Cu}$-bound $\operatorname{PrP}$ has high affinity and catalytic activity for oxidation of some aromatic monoamines such as dopamine which is abundantly present in the neuronal tissues [17, 18]. Among aromatic monoamines tested as the inducers (substrates) for PrP-peptide catalyzed $\mathrm{O}_{2}{ }^{\bullet}$-generating reaction, the most active species were shown to be hydroxylated monoamines such as tyramine (preferably with a hydroxyl group positioned on the aromatic ring with meta- or para-orientation), while non-hydroxylated monoamines such as benzylamine and phenylethylamine were less active [8]. Especially, the action of $\operatorname{PrP}$ helical peptide in combination with tyramine was robust and long lasting (for 10-20 min) while those measured in the presence of benzylamine or phenylethylamine were merely spikes lasting for some seconds.

In the present study, we tested the possibility that amino acid residues on proteins or peptides can be the targets of $\mathrm{Cu}$-bound PrP-mediated oxidative reactions. Here, we compared the effects of 20 amino acids as putative substrates for the $\mathrm{Cu}$-loaded $\operatorname{PrP}$ peptide-catalyzed $\mathrm{O}_{2}{ }^{-}$- generating reaction, since two aromatic amino acids, tyrosine and phenylalanine, are structurally analogous to the highly active substrate tyramine and relatively less active substrate phenylethylamine, respectively; and tryptophan has similarity with inactive monoamine tryptamine. Lastly, effect of PrP-derived Y-rich peptide sequences were tested as putative targets of the PrP-catalyzed oxidative reactions. 
A Generalized PrP structure

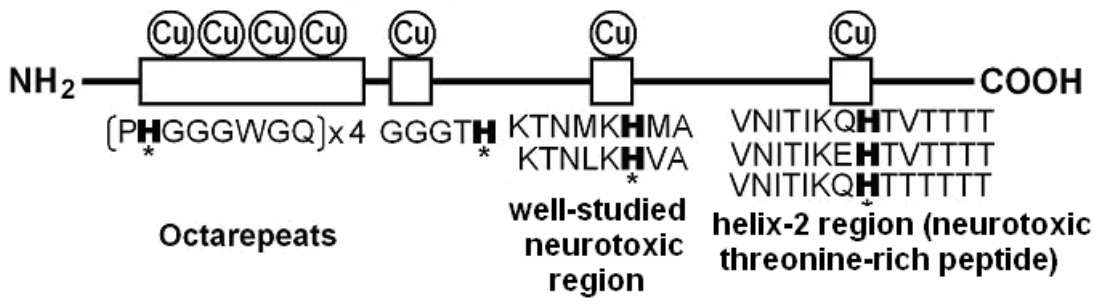

B Possible structure of peptide-Cu complex

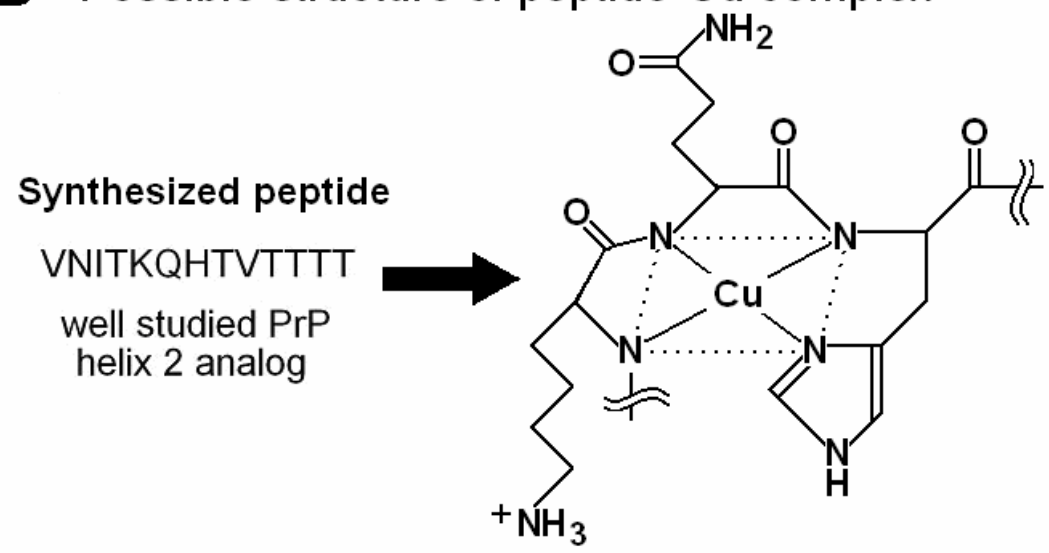<smiles>CCCc1ccc(O)cc1</smiles>

(1)<smiles>NCCc1ccccc1</smiles>

(2)
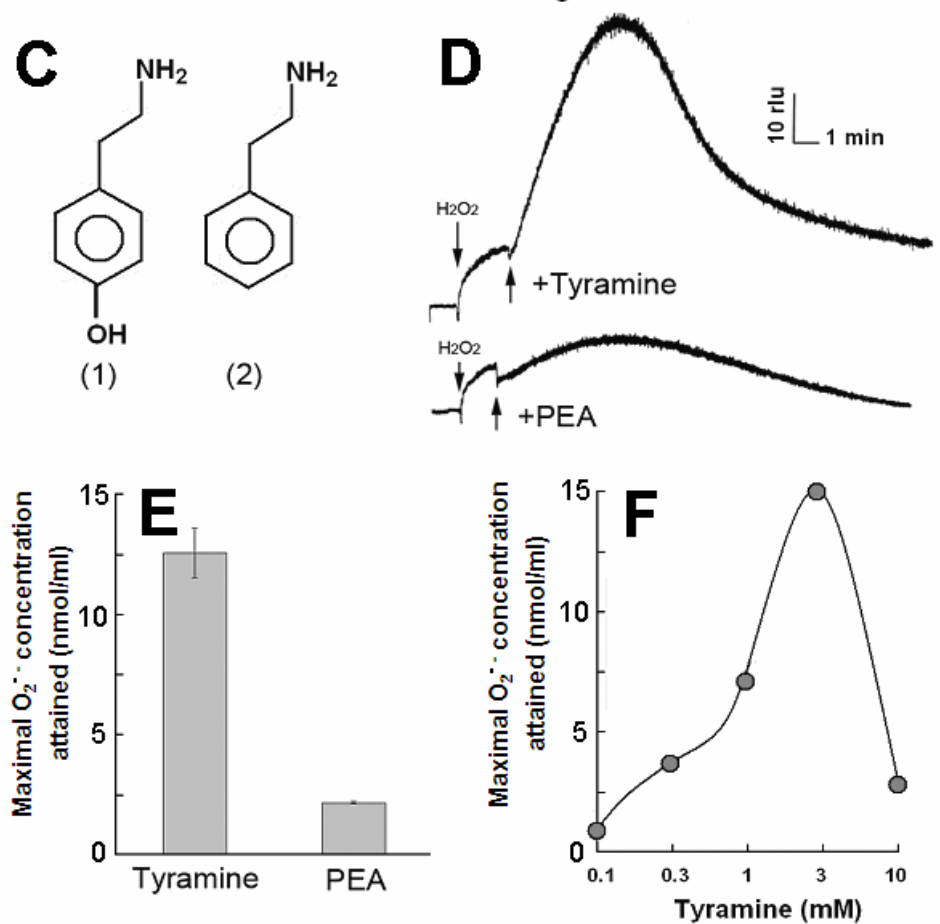

Figure I. PrP-derived Cu-binding peptide catalyzed generation of superoxide in the presence of tyramine and phenyl ethylamine (PEA). (A) Generalized PrP structure. (B) Likely structure of peptide-Cu complex. (C) Chemical structure of (I) tyramine and (2) PEA. (D) Typical curves of tyramine-dependent $\mathrm{O}_{2}{ }^{\circ}$ - production measured as an increase in CLA-chemiluminescence. The observed burst of $\mathrm{O}_{2}{ }^{--}$production in response to tyramine treatment was gradual and long lasting. In contrast, relatively poorer substrate PEA showed minor $\mathrm{O}_{2}{ }^{--}$production. (E) Comparison of the $\mathrm{O}_{2}{ }^{\circ-}$ production in the presence of tyramine and PEA. (F) Effect of tyramine concentration on the $\mathrm{O}_{2}{ }^{--}$production. Complex structure shown in $B$ was estimated according to [2I]. 


\section{Materials and methods}

\section{Chemicals}

The model sequence for threonine-rich Cu-binding region (VNITKQHTVTTTT) in which predicted Cu-binding site are preserved (Fig. 1B), was used as proposed by Brown et al [19], since this sequence was shown to be active as the catalyst for generation of $\mathrm{O}_{2}{ }^{-}$in the presence of some aromatic monoamines $[8,20]$. The likely structure of $\mathrm{Cu}$-peptide complex shown in Fig. 2B was estimated according to [21] and briefly confirmed with the molecular orbital calculations (unpublished results). This model shows that Cu-bound form of VNITKQHTVTTTT is no longer helical but planar, possibly affecting the tertiary structure of PrP.

The catalytic and target peptides chemically synthesized and purified on high pressure liquid chromatography (VNITKQHTVTTTT, purity 99.02\%; DYEDRYYRENMHR, purity 96.30\%; DFEDRFFRENMHR, purity $95.10 \%$; YYR, purity $97.40 \%$ ) were obtained from the custom peptide service department of Sigma Genosis Japan (Ishikari, Hokkaido).

2-Methyl-6-phenyl-3,7-dihydroimidazo[1,2-a]pyrazin -3-one (Cypridina luciferin analog, CLA), a chemiluminescence reagent specific to $\mathrm{O}_{2}{ }^{\bullet-}$, was purchased from Tokyo Kasei Kogyo Co. (Tokyo, Japan). Phenylethylamine, tyramine hydrochloride, tryptamine hydrochloride, tyrosine, phenylalanine, and tryptophan were purchased from Wako Pure Chemical Industries Ltd. (Osaka, Japan). Other chemicals used in this study were of reagent grade purchased from Sigma (St. Louis, MO, USA).

\section{Detection of superoxide}

The peptides and other chemicals were dissolved in phosphate buffer $(\mathrm{pH} \mathrm{7.0)}$ and the generation of $\mathrm{O}_{2}{ }^{*}$ was monitored with the chemiluminescence of CLA with a luminometer (Luminescensor PSN AB-2200-R, Atto Corp., Tokyo, Japan) and the level of $\mathrm{O}_{2}{ }^{*}$ - was expressed with relative luminescence units (rlu) as previously described [22]. CLA-chemiluminescence specifically indicates the generation of $\mathrm{O}_{2}{ }^{\cdot-}$ (and ${ }^{1} \mathrm{O}_{2}$ to a lesser extent) but not that of $\mathrm{H}_{2} \mathrm{O}_{2}$ or $\mathrm{HO}^{\cdot}$ [23]. According to our previous study, the signal for ${ }^{1} \mathrm{O}_{2}$ can be minimized by avoiding the use of high concentration of organic solvents such as ethanol (over $2 \%, v / v)$ in the reaction media [24]. Thus, the induced chemiluminescence recorded here reflects the generation of $\mathrm{O}_{2}{ }^{\circ}$ rather than ${ }^{1} \mathrm{O}_{2}$.

Calibration of CLA-chemiluminescence reflecting the changes in $\mathrm{O}_{2}{ }^{-}$level was carried out by drop- ping the solution of potassium superoxide $\left(\mathrm{KO}_{2}\right)$ dissolved in DMSO onto the CLA containing media as previously reported elsewhere [25].

\section{Results and Discussion}

\section{Oxidative burst catalyzed by the Cu-binding peptide}

Previously, direct interactions between $\mathrm{Cu}$-loaded PrP-derived peptides and some aromatic monoamines leading to generation of $\mathrm{O}_{2}{ }^{*-}$ has been examined in phosphate buffer $(\mathrm{pH}$ 7.0) containing 5 $\mu \mathrm{M}$ CLA, $75 \mu \mathrm{M}$ - $3.0 \mathrm{mM} \mathrm{CuSO}_{4}, 0.3$ - $2.0 \mathrm{mM} \mathrm{H}_{2} \mathrm{O}_{2}$, and $25 \mu \mathrm{M}-0.6 \mathrm{mM}$ helical peptide (or other PrP-derived $\mathrm{Cu}$-binding peptides), by measuring the $\mathrm{O}_{2}{ }^{-}$-specific chemiluminescence [8]. Since requirements for co-factors both copper and $\mathrm{H}_{2} \mathrm{O}_{2}$ were suggested in the previous study, we employed similar standard composition of the reaction mixture (optimized for aromatic amine oxidation) in search for the novel substrates for PrP-mediated peroxidative redox reaction leading to $\mathrm{O}_{2}{ }^{-}$- generation, here.

Molar ratios among the components in the reaction mixture (totally $200 \mu \mathrm{l}$ ), namely catalytic peptide, $\mathrm{Cu}^{2+}, \mathrm{H}_{2} \mathrm{O}_{2}$, and substrates were approximately $1: 3: 3$ : 3 (i.e., each reaction mixture contained $0.15 \mathrm{mM}$ model helical peptide, $0.5 \mathrm{mM} \mathrm{CuSO}_{4}, 0.5 \mathrm{mM} \mathrm{H}_{2} \mathrm{O}_{2}$ and $0.5 \mathrm{mM}$ substrates (monoamines, phenolics, amino acids or target peptides). Fig. $1 \mathrm{C}$ shows the structures of the representative chemicals tested. Generation of $\mathrm{O}_{2}{ }^{--}$was assessed by adding CLA, peptide, $\mathrm{Cu}, \mathrm{H}_{2} \mathrm{O}_{2}$ and substrate molecules to the phosphate buffer in this order.

By adding tyramine to the reaction mixture as a model substrate, typical curves of $\mathrm{O}_{2}{ }^{*-}$ production could be observed as the increase in CLA-chemiluminescence (Fig. 1D-F). The observed burst of $\mathrm{O}_{2}{ }^{-}$production in response to tyramine treatment was gradual and long lasting at high level.

In contrast, relatively poorer substrate phenylethylamine showed less production of $\mathrm{O}_{2}{ }^{-}$at $0.5 \mathrm{mM}$ (Fig. 1D,E). For showing the effect of phenylethylamine as a pro-oxidant in the present system, much higher concentrations $(>3 \mathrm{mM})$ were requires as previously reported [8] (data not shown). Above data suggest that the Cu-peptide complex recognizes the key structural difference among the substrates sharing similar structural backbones. In this case, the only difference between the active and the poor substrates was the presence of $\mathrm{OH}$ group on the aromatic moiety (thus possibly preferring phenolic moiety). In addition, tryptamine was also tested and shown to be poorer substrate (Fig. 3A). 
<smiles>NC(Cc1ccc(O)cc1)C(=O)O</smiles>

B
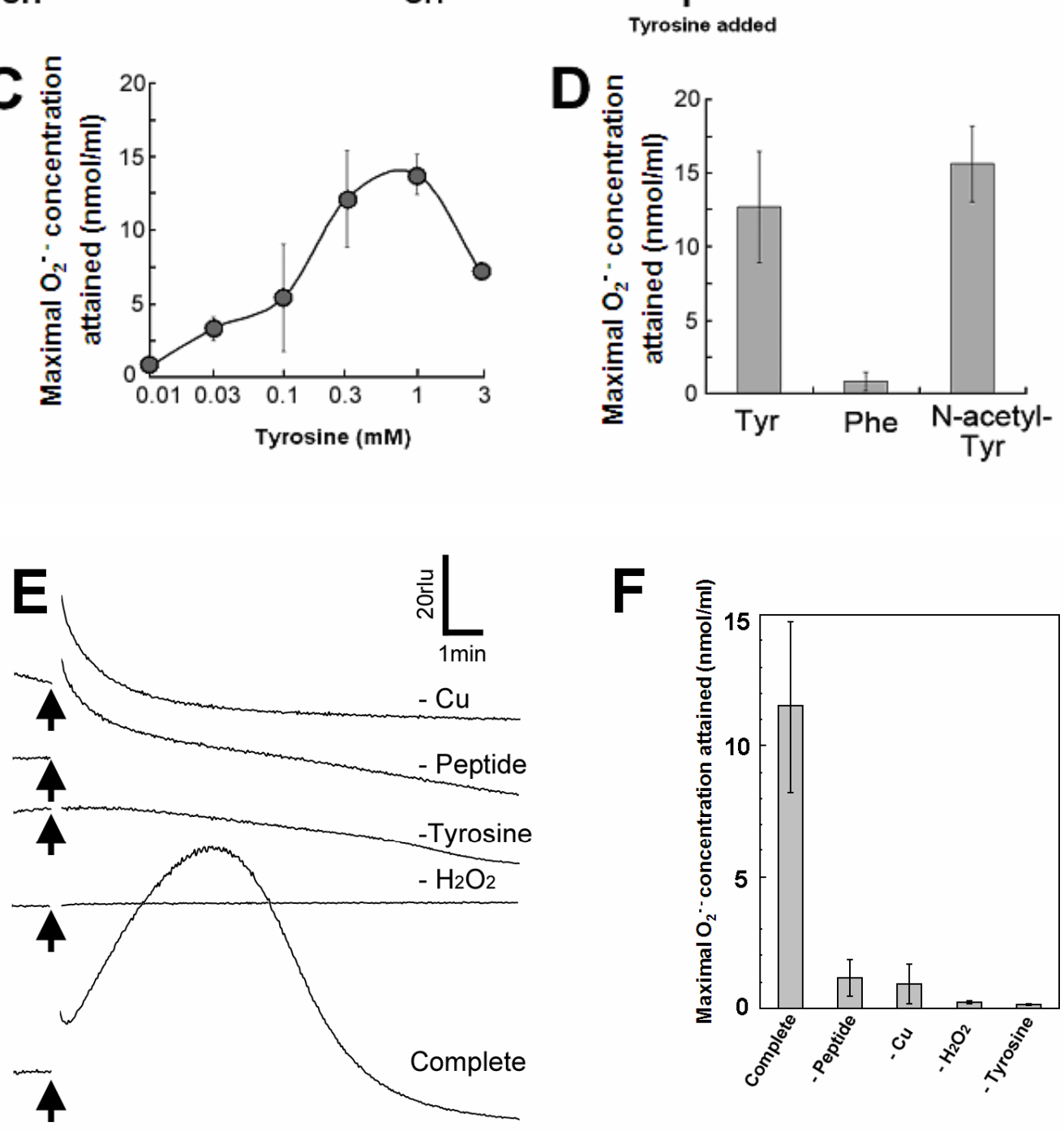

Figure 2. Aromatic amino acids as putative substrates for superoxide-generating reaction. (A) Chemical structure of the substrates tested, (3) tyrosine, (4) phenylalanine, (5) N-acetyl-tyrosine. (B) Typical trace of the tyrosine-induced production of $\mathrm{O}_{2}{ }^{-}$. (C) Effect of tyrosine concentration on the production of $\mathrm{O}_{2}{ }^{-}$. (D) Comparison of three structurally related amino acids as the substrates for $\mathrm{O}_{2}{ }^{-}$-producing reaction. These data imply that neither of carboxyl group nor amino group was the key group necessarily required in the active substrates. (E) Requirement of the co-factors, substrate and catalytic peptide for generation of $\mathrm{O}_{2}{ }^{-}$. (F) Comparison of $\mathrm{O}_{2}{ }^{-}$- production in the presence and absence of key components. Means of triplicates are shown in (C), (D) and (F). Error bars in (C), (D), (F) represent S.E. 

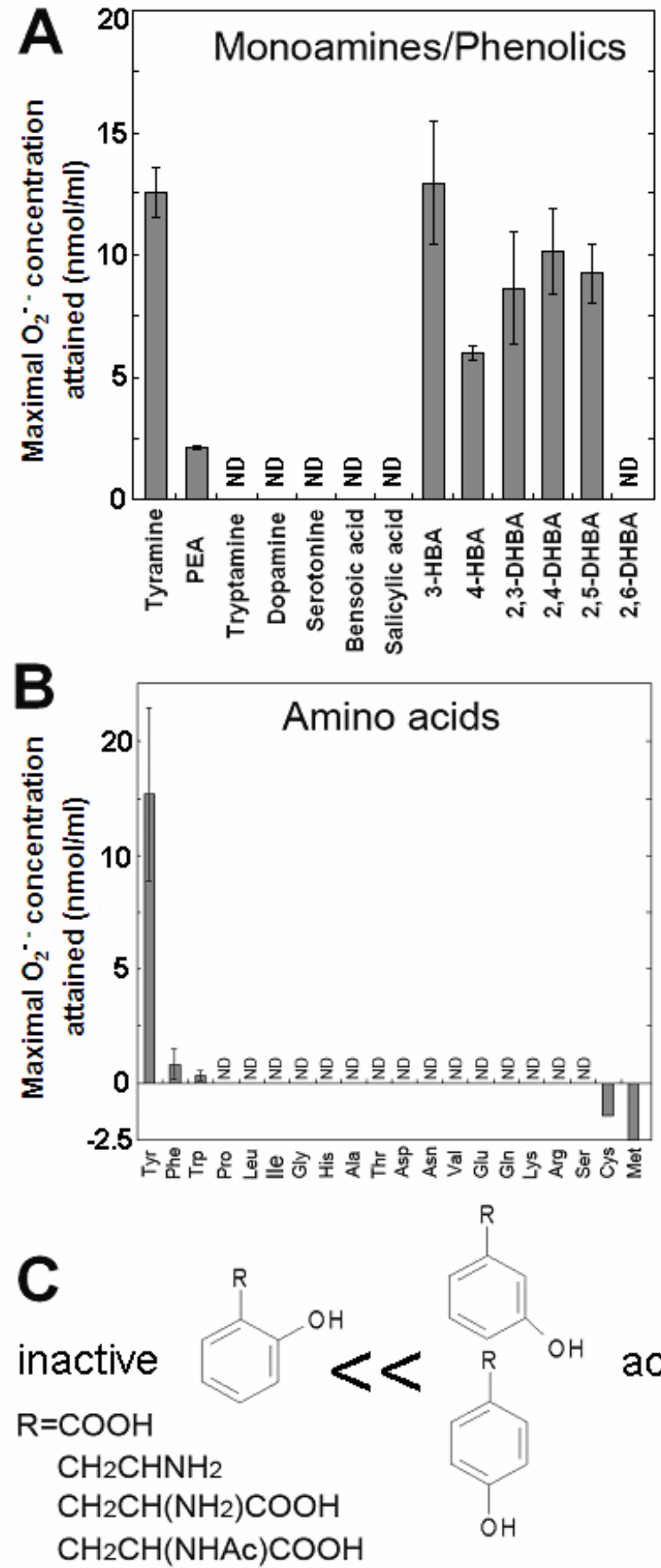

Figure 3. Effects of various monoamines, phenolics and amino acids on the superoxide-generating reactions catalyzed by the Cu-bound helical PrP peptide. (A) Effects of various monoamines and phenolics. (B) Effects of 20 natural amino acids. (C) Structural features of good substrates. Means of triplicates are shown. Error bars, S.E.

\section{Generation of $\mathrm{O}_{2}{ }^{--}$in the presence of tyrosine}

By structural similarity to aromatic monoamines, we tested the effects of aromatic amino acids and related compounds, namely tyrosine, phenylalanine and N-acetyl-tyrosine, as the putative substrates (Fig. $2 \mathrm{~A}$, compounds $3,4,5$ respectively). In order to examine the effect of tyrosine (as an analog of tyramine) on the generation of $\mathrm{O}_{2}{ }^{\bullet-}, 0.5 \mathrm{mM}$ tyrosine was added to the reaction mixture with identical compositions as described above and the induced increase in CLA chemiluminescence was monitored (Fig. 2B).

Effect of tyrosine concentration on the generation of $\mathrm{O}_{2}{ }^{-}$is shown in Fig. 2C. The optimal concentration was shown to be $c a .0 .3-1 \mathrm{mM}$. By comparing with the standard curve for $\mathrm{KO}_{2}$, the concentrations of $\mathrm{O}_{2}{ }^{-}$generated in the reaction mixture in response to addition of $0.5 \mathrm{mM}$ tyrosine and tyramine were estimated (Fig. 2D). In contrast, addition of phenylalanine resulted in no drastic increase in CLA-chemiluminescence (Fig. 2D). Here again, the only difference between two substrates with largely differed in their activity was merely the presence of $\mathrm{OH}$ group on the aromatic ring. In addition, tryptophan was shown to be much poorer substrate (Fig. 3B) as expected from the behaviors of structurally similar monoamines such as tryptamine and serotonin (Fig. 3A). Similarly to the previous report [8], importance of co-factors namely $\mathrm{H}_{2} \mathrm{O}_{2}$ and $\mathrm{Cu}$ in tyrosine-dependent production of $\mathrm{O}_{2}{ }^{*}$ - was confirmed (Fig. 2E,F). Reaction mixtures lacking either of two co-factors, substrate (tyrosine) or catalytic peptide showed no increase in $\mathrm{O}_{2}{ }^{\bullet-}$ generation.

S-containing amino acids, namely, Met and Cys lowered the background level of chemiluminescence and no induction of oxidative burst was measured (Fig. 3B). Effects of other amino acids (all non-aromatic; Ala, Arg, Asn, Asp, Gln, Glu, Gly, His, Ile, Leu, Lys, Pro, Ser, Val, and Thr) were also examined and none of them induced the production of $\mathrm{O}_{2}{ }^{--}$ (Fig. 3B). These data imply that neither of carboxyl group nor amino group was the key group necessarily required in the active substrates. This was confirmed with the use of N-acetylated tyrosine in which amino group is masked with acetyl group. The activity of $\mathrm{N}$-acetyl tyrosine was as high as of tyrosine and tyramine (Fig. 2D). Since tyramine lacks carboxylic group, no involvement of carboxylic moiety in these active substrates is obvious.

Since the importance of phenolic moieties in tyrosine and tyramine were suggested, we examined the effects of various phenolics (some examples of the 
hydroxylated benzoates are shown in Fig. 3A). Data suggested the importance of meta- and para-positioned $\mathrm{OH}$ group on aromatic rings. Presence of ortho-positioned $\mathrm{OH}$ group was not inhibitory only if there was additional $\mathrm{OH}$ group positioned in meta- or para-orientations as observed with 2,3-, 2,4- and 2,5-dihydroxybenzoic acids (Fig. 3A). In contrast, no oxidative activity was observed with (1) non-phenolic compound such as benzoate nor (2) phenolics (and catecholamines) bearing $\mathrm{OH}$ group at ortho-position(s) such as salicylic acid, 2,6-dihydroxybenzoic acid and dopamine. These data suggest that the action of $\mathrm{Cu}$-bound PrP-derived peptides are similar to the phenol-oxidizing enzymes.

\section{Tyrosine-rich peptide sequences as possible targets}

Above study revealed that the common structural feature required to be active substrates for $\mathrm{Cu}$-bound $\operatorname{PrP}$ was shown to be the presence of phenolic moiety as illustrated in Fig. 3C. Thus, in case of tyrosine, the presence of phenolic moiety, but not the $-\mathrm{NH}_{2}$ or $-\mathrm{COOH}$ groups was shown to be important in interaction with $\mathrm{Cu}$-loaded PrP peptide. Therefore, it is natural to consider the tyrosine residues on peptides or proteins as putative targets of this type of reaction.

Recent reports suggested that helix $\mathrm{H} 1$ of $\mathrm{PrP}$ and its two flanking loops (highly rich in tyrosine residues) undergo a conformational transition into a $\beta$-like structure during conformational conversion of the $\mathrm{PrPC}$ into PrPsc, which is a fundamental event in the onset of TSE [26]. In this section, we describe our attempt to test if tyrosine residues on PrP or derived peptides, in addition to free tyrosine, can be the putative targets of $\mathrm{Cu}$-loaded catalytic PrP peptide. Firstly, we examined the effect of a tyrosine-containing tripeptide sequence (tyrosyl-tyrosyl-arginine, YYR) which can be found two times in the PrP's Y-rich region (DYEDR-YYR-ENMHRYPNQV-YYR-PMDEY) as a potent inducer (substrate) of $\mathrm{O}_{2}{ }^{-}$-generating reaction catalyzed by $\mathrm{Cu}$-bound catalytic peptide (Fig. 4). Such duplicated YYR motifs are conserved in bovine, goat, human and pig PrPs. Fig. 4A is showing the typical trace of YYR-induced $\mathrm{O}_{2}{ }^{*-}$ generation catalyzed by the Cu-loaded and $\mathrm{H}_{2} \mathrm{O}_{2}$-fueled VNITKQHTVTTTT. Compared to free tyrosine, YYR was shown to be much more active as oxidative burst inducers (Fig. 4B). These data indicate that both free form and peptide-integrated forms of tyrosine can be recognized by the $\mathrm{Cu}$-loaded catalytic peptide.

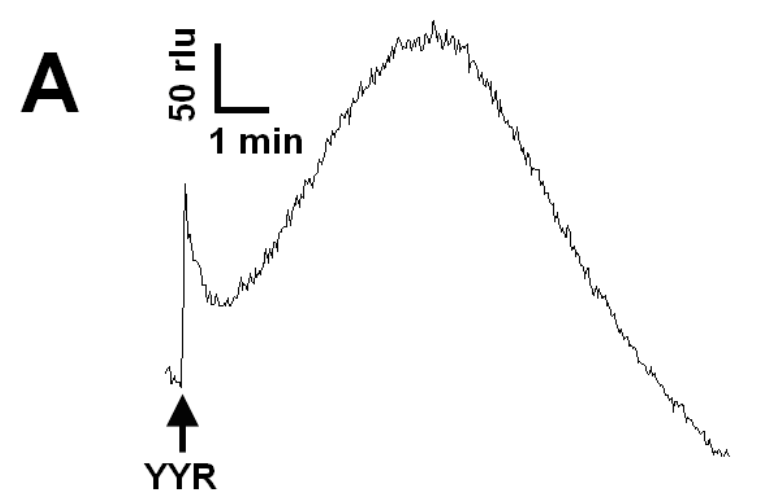

B

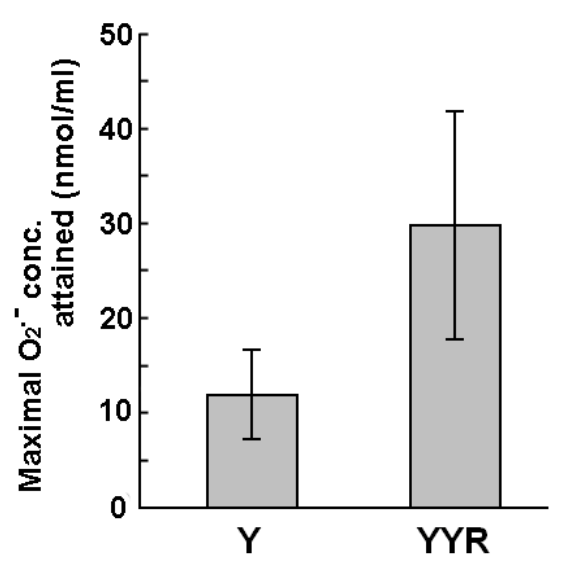

Figure 4. Effect of tyrosyl-tyrosyl-arginine as putative target of the Cu-bound PrP helical peptide-catalyzed reaction. (A) Typical trace of CLA-chemiluminescence in the presence of $0.5 \mathrm{mM}$ YYR peptide. (B) Comparison of the effects of free tyrosine $(0.5$ $\mathrm{mM})$ and YYR peptide $(0.5 \mathrm{mM})$. Means of triplicates are shown. Error bars, S.E.

Lastly, reactivities of longer peptide sequences corresponding to the Y-rich region in $\operatorname{PrP}$ (D $\underline{Y E D R Y Y R E N M H R) ~ o r ~ i t s ~ Y-t o-F ~ s u b s t i t u t i o n ~ m u-~}$ tant (DFEDRFFRENMHR) to the Cu-loaded catalytic peptide were tested (Fig. 5). Peptide solutions were added to the model reaction mixture containing the catalytic peptide and co-factors (identical composition as above experiments). Since addition of longer peptides often results in lowering of basal chemiluminescence level compared to the cases of free amino acids or short oligo-peptides (data not shown), the level of chemiluminescence increase following addition of Y-rich peptide (D $\underline{Y E D R Y Y R E N M H R) ~ w a s ~ n o t ~}$ as great as we expected. However, when compared with the Y-to-F substitution mutant sequence, the level of $\mathrm{O}_{2}{ }^{--}$production with PrP-derived native Y-rich sequence was obviously higher (Fig. 5). This suggests that the tyrosine residues on intra-, and inter-PrP molecules could be additional target of the $\mathrm{Cu}$-bound PrP-catalyzed reaction. 


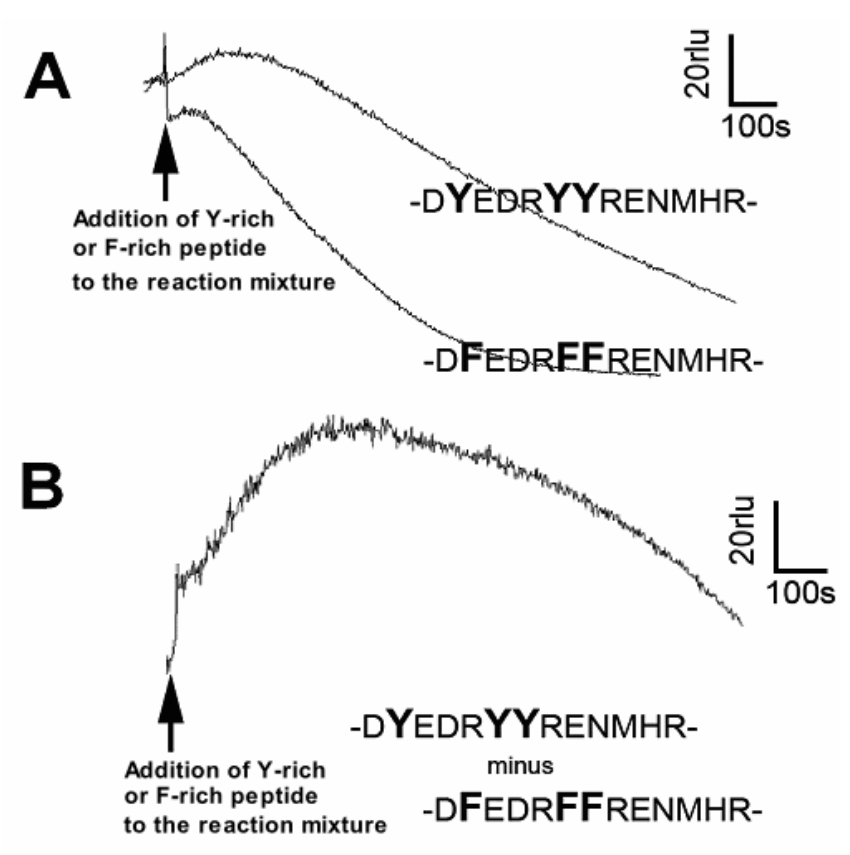

Figure 5. Effect of tyrosine-rich sequence in human PrP as putative target of the Cu-bound PrP-catalyzed reaction. (A) Typical traces of CLA-chemiluminescence in the presence of natural Y-rich peptide and Y-F substituted mutant peptide. (B) Synthesized trace made by subtracting the curve for mutant peptide from that for Y-rich peptide.

Recently a French group conducted a study with synthetic sheep PrP peptides corresponding to native sequence spanning helix 1 and $\beta$-strand 2 (residues 142-166; corresponding to the Y-rich region), and their mutant sequences such as R156A mutant (YYR $\rightarrow$ YYA) and the Y155A mutant (YYR $\rightarrow$ YAR), and revealed that tyrosine 155 plays a key role in the stabilization of the beta-hairpin-like conformation of the sheep peptide [26], suggesting that YYR motifs are involved in the early event of conformational changes. Furthermore, drastic changes in the 3-dimentional localization of YYR domain in Y-rich region within $\operatorname{PrP}$ molecule were suggested. Recent reports suggested that YYR sequence in PrPs can be susceptible to the redox changes including labeling by nitrating reagents, if the residues were facing the hydrophilic surface of the PrP protein globule. According to Lennon et al. [27], two conserved tyrosine residues on hamster $\operatorname{PrP}$ (tyrosines 149 and 150) are insensitive to labeling by nitrating reagents, if $\mathrm{PrP}$ was in the normal cellular form. However, these tyrosine residues become reactive after the protein has been converted to the $\beta$-oligomeric isoform. There is another pair of tyrosine residues placed next to each other in the C-terminal region in mammalian PrPs (Y225, 226Y in human PrP; 236, Y237 in bovine PrP). Interestingly, the C-terminal $Y Y$ is facing the hydrophilic surface in the $\operatorname{PrPC}$ and hidden by the hydrophobic packaging in PrPSc [27]. Thus, behavior of specific tyrosine residues (susceptible to nitration) can be a hallmark of conversion of normal PrP into a structure that is enriched in beta-sheet.

\section{Requirement for co-factors}

In the reaction mixtures lacking either of 4 components, namely helical peptide, $\mathrm{Cu}, \mathrm{H}_{2} \mathrm{O}_{2}$ and tyrosine, optimal level of the $\mathrm{O}_{2}{ }^{*}$-dependent chemiluminescence could not be achieved (Fig. 2E,F). This suggests that any component is certainly required for the reaction leading to generation of $\mathrm{O}_{2}{ }^{\circ}$. Each of 4 components involved in the reaction acted in the concentration-dependent manners, further confirming that these components are necessarily involved in the $\mathrm{O}_{2}{ }^{-}$-generating reaction and thus supplies of each component can be the limiting factors (data not shown). Since the requirement for $\mathrm{H}_{2} \mathrm{O}_{2}$ was confirmed here, the type of reaction catalyzed by the $\mathrm{Cu}$-supplemented $\operatorname{PrP}$ helical sequence is rather similar to the aromatic monoamine-oxidizing and/or the phenol-oxidizing reactions leading to $\mathrm{O}_{2}{ }^{-}$generation known to be catalyzed by $\mathrm{H}_{2} \mathrm{O}_{2}$-requiring plant peroxidases [28-30] and human methemoglobin [31]. The proposed reaction in which the $\mathrm{Cu}$-loaded helix converts the molecular oxygen into $\mathrm{O}_{2}{ }^{\bullet}$, is fueled by $\mathrm{H}_{2} \mathrm{O}_{2}$ and bio-phenolics. We can expect that supply of $\mathrm{H}_{2} \mathrm{O}_{2}$ can take place within the $\operatorname{PrP}$ molecule since another $\mathrm{Cu}$-binding region in PrP known as the neurotoxic region ( $\mathrm{PrP} 106-126)$ reportedly catalyzes the production of $\mathrm{H}_{2} \mathrm{O}_{2}$ [13].

\section{Possible mechanism of superoxide generation}

The present study showed that phenolics including tyrosine and tyramine (to lesser extent with non-phenolic aromatic monoamines) were shown to be active substrates for generation of $\mathrm{O}_{2}{ }^{\bullet}$. Since $\mathrm{H}_{2} \mathrm{O}_{2}$ is required for initiation of the reaction, the type of reaction studied here is distinct from the reactions catalysed by known Cu-centered enzymes such as $\mathrm{Cu}$-amine oxidase, phenol oxidase and tyrosinase. Our earlier works revealed that 1-electron oxidation of phenolics and aromatic monoamines by plant peroxidase and human methemoglobin (pseudoperoxidase reaction), results in generation of $\mathrm{O}_{2} \cdot-[25,28-31]$. In cases of phenolics, generation of phenoxy radicals (detectable with electron spin resonance using ascorbate as a spin trapping agent) was suggested as an redox intermediate leading to reduction of molecular oxygen to form $\mathrm{O}_{2}{ }^{\circ}$. Conversion of heme proteins 
such as hemoglobin and cytochrome $c$ into peroxidase-like catalysts through protein inactivation or degradation is well studied. One recent study revealed that Amyloid- $\beta$ peptide incorporating heme can be converted to be a peroxidase-like protein [32]. This work elegantly related the peroxidase-like reaction and the cytopathogenesis of Alzheimer's disease which is a typical protein conformational disease sharing great similarity with prion dementias. By this way, it is likely that the protein with no peroxidase activity can be converted to a peroxidase-like catalyst upon binding to catalytically active complex (such as heme) or metals (such as $\mathrm{Cu}$ ).

By analogy to the (pseudo)peroxidase models, we hypothesize that the mechanism for $\mathrm{Cu}$-bound PrP-catalyzed $\mathrm{O}_{2}{ }^{*-}$ generating reaction is mediated with peroxidase-type 1-electron oxidation of phenolics followed by formation of phenoxy radicals.

\section{Activity of other Cu-binding sequence}

Our present study focused on the behavior of model PrP helix with Cu-binding motif as inspired by earlier work conducted by Brown et al [19]. In addition to the model helical peptide, activities of native helical sequence (VNITIKQHTVTTTT) and other cupper-binding motifs (PHGGGWGQ, GGGTH, and KTNMKHMA) on PrPs were also examined in our preliminary tests (data not shown). We have confirmed that all Cu-binding motifs except for KTNMKHMA showed the catalytic activities for tyrosine. Therefore detailed analysis of the action of other Cu-binding motifs in PrP towards tyrosine and tyrosine-rich peptides must be examined in the future research effort.

\section{Possible role of tyrosine-dependent reaction}

According to the model proposed by Lee and Eisenberg [33], there may be an intermediate structure of $\operatorname{PrP}$ between $\operatorname{PrPC}$ and $\mathrm{PrPs}$, to be formed from $\mathrm{PrPC}^{\mathrm{C}}$ in response to cellular redox changes (thus designated as $\operatorname{PrPRDX}$ ). An in vitro study demonstrated that $\operatorname{PrPRDX}$ of recombinant hamster PrP is catalytically active and capable of further conversion of $\mathrm{PrPC}$ to $\operatorname{PrPRDX}$. At the end, PrPRDX accumulates and disulfide bonds are formed among PrPRDX molecules, and lastly formation of $\mathrm{PrPSc}$ is completed. This attractive model explains how pathogenic form of $\operatorname{PrP}$ could be propagated by the presence of an intermediate PrPs. However, this model lacks the view on the very initial event possibly occurring on a single molecule as discussed in our previous article [8]. Kawano has proposed that PrP-catalyzed generation of $\mathrm{O}_{2}{ }^{*-}$ (that may attack the $\mathrm{SH}$ residues on $\mathrm{PrP}$ ) may stimulate the conversion of monomeric native $\mathrm{PrPC}$ to the catalytically active PrPRDX intermediate which in turn acts as the seed for further production of PrPRDX from PrPC [8], based on the report by Shiraishi et al. [18] supporting the view in which the $\mathrm{Cu}$ bound to $\mathrm{PrP}$ undergoes catalytic cycling in the presence of catecholamines and causes the oxidation of protein, possibly involving the ROS-generating reactions. The model with involvement of catecholamines and related chemicals (including their precursors and/or analogues) is very attractive since such chemicals are highly rich in brain and neuronal tissues. In addition to such monoamines, the present work focused on the role of amino acids as alternative substrates for the PrP-catalyzed oxidative burst and tyrosine was shown to be the best and only substrate among all amino acids tested. Tyrosine was shown to be capable of inducing long lasting and robust production of $\mathrm{O}_{2}{ }^{*-}$ in the reaction mixture containing both $\mathrm{H}_{2} \mathrm{O}_{2}$ and $\mathrm{Cu}$-loaded PrP helix-2 model peptide. Since tyrosine is much more abundant throughout the tissues and cells compared to catecholamine-related signaling molecules, this reaction is much more likely to take place in the prion biology.

In addition to the action of free tyrosine, effect of two tyrosine-rich peptide sequences $\underline{Y Y R}$ and DYEDRYYRENMHR found in human $\operatorname{PrP}$ corresponding to the $Y$-rich region (DYEDRYYRENMHRYPNQVYYRPMDEY) was tested as putative substrates for the threonine-rich neurotoxic peptide loaded with $\mathrm{Cu}$ and $\mathrm{H}_{2} \mathrm{O}_{2}$. YYR motif found twice in the Y-rich region was shown to be highly active substrate for the reaction. Comparison of Y-rich sequence consisted of 13 amino acids and its Y-to-F substitution mutant sequence revealed that the tyrosine-residues on Y-rich peptide derived from PrP may contribute to the higher production of $\mathrm{O}_{2}{ }^{-}$- in the presence of Cu-bound, $\mathrm{H}_{2} \mathrm{O}_{2}$-fuelled catalytic peptide. These data suggest that the tyrosine residues on PrP molecules could be additional targets of the PrP-mediated reactions through intra- or inter-molecular interactions. In the future works, impacts of such redox events on the conformational changes in PrP must be clarified.

\section{Acknowledgments}

This work was supported by a grant of Knowledge Cluster Initiative implemented by Ministry of Education, Culture, Sports, Science and Technology (MEXT), Japan.

\section{Abbreviations}

CLA: Cypridina luciferin analog; $\mathrm{HO}^{\bullet}$ : hydroxyl radicals; $\mathrm{O}_{2} \cdots$ : superoxide anion; $\mathrm{PrP}$ : prion protein; 
PrPC: intrinsic cellular PrP; PrPsc: scrapie isoform of PrP; PrPRDX: PrP redox intermediate; rlu: relative luminescence units; ROS: reactive oxygen species; SOD: superoxide dismutase.

\section{Conflict of Interest}

The authors have declared that no conflict of interest exists.

\section{References}

1. Jeffray M, McGovern G, Goodsir CM, et al. Sites of prion protein accumulation in scrapie-infected mouse spleen revealed by immuno-electron microscopy. J Pathol. 2000; 191: 323-32.

2. Tabner BJ, Turnbull S, El-Agnaf $\mathrm{O}$, et al. Production of reactive oxygen species from aggregating proteins implicated in Alzheimer's disease, Parkinson's disease and other neurodegenerative diseases. Curr Top Med Chem. 2001; 1: 507-17.

3. Tabner BJ, El-Agnaf OMA, Turnbull S, et al. Hydrogen peroxide is generated during the very early stages of aggregation of the amyloid peptides implicated in Alzheimer's disease and familial British dementia. J Biol Chem. 2005; 280: 35789-92.

4. Watt NT, Taylor DR, Gillott A, et al. Reactive oxygen species-mediated $\beta$-cleavage of the prion protein in the cellular response to oxidative stress. J Biol Chem. 2005; 280: 35914-35921.

5. Wong BS, Brown DR, Pan T, et al. Oxidative impairment in scrapie-infected mice is associated with brain metals perturbations and altered antioxidant activities. J Neurochem. 2001; 79: 689-98.

6. Sauer H, Dagdanova A, Hescheler J, et al. Redox-regulation of intrinsic prion expression in multicellular prostate tumor spheroids. Free Radic Biol Med. 1999; 27: 1276-83.

7. Opazo C, Barria MI, Ruiz FH, et al. Copper reduction by copper binding proteins and its relation to neurodegenerative diseases. Biometals. 2003; 16: 91-8.

8. Kawano T. Prion-derived copper-binding peptide fragments catalyze the generation of superoxide anion in the presence of aromatic monoamines. Int J Biol Sci. 2007; 3: 57-63.

9. Furuichi T, Kawano T. Possible application of electron spin resonance to monitoring of prion diseases and hypotheses on oxidative action and propagation of copper-bound infectious protein. Bull Nippon Sport Sci Univ. 2005; 35: 71-80.

10. Kawano T. Quenching and enhancement of terbium fluorescence in the presence of prion-derived copper-binding peptides. ITE Lett. 2006; 7: 383-5.

11. Ronga L, Palladino P, Saviano G, et al. Structural characterization of a neurotoxic threonine-rich peptide corresponding to the human prion protein alpha2-helical 180-195 segment, and comparison with full-length alpha2-helix-derived peptides. J Pept Sci. 2008;14:1096-102.

12. Tabner BJ, Turnbull S, Fullwood NJ, et al. The production of hydrogen peroxide during early-stage protein aggregation: a common pathological mechanism in different neurodegenerative diseases? Biochem Soc Trans. 2005; 33: 548-50.

13. Turnbull S, Tabner BJ, Brown DR, et al. Copper-dependent generation of hydrogen peroxide from the toxic prion protein fragment PrP106-126. Neurosci Lett. 2003; 336: 159-62.

14. Turnbull S, Tabner BJ, Brown DR, et al. Generation of hydrogen peroxide from mutant forms of the prion protein fragment PrP 121-231. Biochemistry. 2003; 42: 7675-81.
15. Agostinho $P$, Oliveira CR. Involvement of calcineurin in the neurotoxic effects induced by amyloid- $\beta$ and prion peptides. Eur J Neurosci. 2003; 17: 1189-96.

16. Belosi B, Gaggelli E, Guerrini R, et al. Copper binding to the neurotoxic peptide PrP106-126: thermodynamic and structural studies. Chembiochem. 2004; 5: 349-59.

17. Shiraishi N, and Nishikimi M. Carbonyl formation on a copper-bound prion protein fragment, PrP23-98, associated with its dopamine oxidase activity. FEBS Lett. 2002; 511: 118-22.

18. Shiraishi N, Inai $Y, B i ~ W$, et al. Fragmentation and dimerization of copper-loaded prion protein by copper-catalysed oxidation. Biochem J. 2005; 387: 247-55.

19. Brown DR, Guantieri V, Grasso G, et al. Copper(II) complexes of peptide fragments of the prion protein. Conformation changes induced by copper(II) and the binding motif in C-terminal protein region. J Inorg Biochem. 2004; 98: 133-43.

20. Yokawa K, Kagenishi T, Kawano T. Use of Cypridina luciferin analog for assessing the monoamine oxidase-like superoxide-generating activities of two peptide sequences corresponding to copper-binding motif in human prion and its model analog. In: Kricka LJ, Stanley PE, eds. Bioluminescence and Chemiluminescence. Singapore: World Sci Publ. 2008.

21. Fang YY, Ray BD, Claussen CA, et al. Ni(II).Arg-Gly-His-DNA interactions: investigation into the basis for minor-groove binding and recognition. J Am Chem Soc. 2004; 126: 5403-12.

22. Kawano T, Kawano N, Hosoya H, et al. Fungal auxin antagonist hypaphorine competitively inhibits indole-3-acetic acid-dependent superoxide generation by horseradish peroxidase. Biochem Biophys Res Commun. 2001; 288: 546-51.

23. Nakano M, Sugioka K, Ushijima Y, et al. Chemiluminescence probe with Cypridina luciferin analog, 2-methyl-6-phenyl-3,7-dihydroimidazo[1,2-a]pyrazin-3-one, for estimating the ability of human granulocytes to generate $\mathrm{O}_{2}$. Anal Biochem. 1986; 159: 363-9.

24. Yokawa K, Suzuki N, Kawano T. Ethanol-enhanced singlet oxygen-dependent chemiluminescence interferes with the monitoring of biochemical superoxide generation with a chemiluminescence probe, Cypridina luciferin analog. ITE Lett. 2004; 5: 49-52.

25. Kawano T, Sahashi N, Takahashi K, et al. Salicylic acid induces extracellular superoxide generation followed by an increase in cytosolic calcium ion in tobacco suspension culture: The earliest events in salicylic acid signal transduction. Plant Cell Physiol. 1998; 39: 721-30.

26. Bertho G, Bouvier G, Hoa GH, et al. The key-role of tyrosine 155 in the mechanism of prion transconformation as highlighted by a study of sheep mutant peptides. Peptides. 2008; 29:1073-84.

27. Lennon CW, Cox HD, Hennelly SP, et al. Probing structural differences in prion protein isoforms by tyrosine nitration. Biochemistry. 2007; 46:4850-60.

28. Kawano T. Roles of the reactive oxygen species-generating peroxidase reactions in plant defense and growth induction. Plant Cell Rep. 2003; 2: 829-37.

29. Kawano T, Pinontoan R, Uozumi N, et al. Aromatic monoamine-induced immediate oxidative burst leading to an increase in cytosolic $\mathrm{Ca}^{2+}$ concentration in tobacco suspension culture. Plant Cell Physiol. 2000; 41: 1251-8.

30. Kawano T, Pinontoan R, Uozumi N, et al. Phenylethylamine-induced generation of reactive oxygen species and ascorbate free radicals in tobacco suspension culture: mechanism for oxidative burst mediating $\mathrm{Ca}^{2+}$ influx. Plant Cell Physiol. 2000; 41: 1259-66.

31. Kawano T, Pinontoan R, Hosoya $H$, et al. Monoamine-dependent production of reactive oxygen species catalyzed by pseudoperoxidase activity of human hemoglobin. Biosci Biotechnol Biochem. 2002; 66: 1224-32. 
32. Atamna H, Boyle K. Amyloid- $\beta$ peptide binds with heme to form a peroxidase: Relationship to the cytopathologies of Alzheimer's disease. Proc Natl Acad Sci USA. 2006; 103: 3381-6.

33. Lee S, Eisenberg D. Seeded conversion of recombinant prion protein to a disulfide-bonded oligomer by a reduction-oxidation process. Nat Struct Biol. 2003; 10: 725-30. 